suffice to mediate inflammatory lung disease remains to be elucidated, but platelet-derived mediators may underlie or contribute to the development of ARDS in neutropenic patients.

The notion of platelet-neutrophilic interactions closes a previous conceptual gap in the pathogenesis of ALI and ARDS: it explains the critical relevance of selectins in a setting where the initial retention of leukocytes is predominantly attributable to mechanical factors. Thus, a new role for selectins, not as mediators of leukocyteendothelial cell interaction, but as amplifiers of platelet and leukocyte activation, has emerged in inflammatory lung disease.

Address correspondence to: Wolfgang M. Kuebler, Institute of Physiology, Charité Universitaetsmedizin Berlin, Campus Benjamin Franklin, Arnimallee 22, 14195 Berlin, Germany. Phone: 49-0-30-8445-1648; Fax: 49-0-30-8445-1634; E-mail: wolfgang. kuebler@charite.de.

1. The Acute Respiratory Distress Syndrome Network. 2000. Ventilation with lower tidal volumes as compared with traditional tidal volumes for acute lung injury and the acute respiratory distress syndrome. N. Engl. J. Med. 342:1301-1308.

2. Rubenfeld, G.D., et al. 2005. Incidence and outcomes of acute lung injury. N. Engl. J. Med. 353:1685-1693.

3. Bachofen, M., and Weibel, E.R. 1977. Alterations of the gas exchange apparatus in adult respiratory insufficiency associated with septicemia. Am. Rev. Respir. Dis. 116:589-615.

4. Pittet, J.F., Mackersie, R.C., Martin, T.R., and Matthay, M.A. 1997. Biological markers of acute lung injury: prognostic and pathogenetic significance. Am. J. Respir. Crit. Care Med. 155:1187-1205.

5. Laufe, M.D., Simon, R.H., Flint, A., and Keller, J.B. 1986. Adult respiratory distress syndrome in neutropenic patients. Am. J. Med. 80:1022-1026.

6. Downey, G.P., Worthen, G.S., Henson, P.M., and Hyde, D.M. 1993. Neutrophil sequestration and migration in localized pulmonary inflammation. Capillary localization and migration across the interalveolar septum. Am. Rev. Respir. Dis. 147:168-176.

7. Lien, D.C., et al. 1987. Physiological neutrophil sequestration in the lung: visual evidence for localization in capillaries. J. Appl. Physiol. 62:1236-1243.

8. Kuebler, W.M., Kuhnle, G.E., Groh, J., and Goetz, A.E. 1994. Leukocyte kinetics in pulmonary microcirculation: intravital fluorescence microscopic study. J. Appl. Physiol. 76:65-71.

9. Gebb, S.A., et al. 1995. Sites of leukocyte sequestration in the pulmonary microcirculation. J. Appl. Physiol. 79:493-497.

10. Doerschuk, C.M. 2001. Mechanisms of leukocyte sequestration in inflamed lungs. Microcirculation. 8:71-88.

11. Bock, D., Aydt, E.M., Kuebler, W.M., and Wolff, G. 2006. The role of selectins during lung inflam- mation and their potential impact for innovative therapeutic strategies. Current Respiratory Medicine Reviews. 2:339-354.

12. Zarbock, A., Singbartl, K., and Ley, K. 2006. Complete reversal of acid-induced acute lung injury by blocking of platelet-neutrophil aggregation. J. Clin. Invest. 116:3211-3219. doi:10.1172/JCI29499.

13. Kiefmann, R., Heckel, K., Schenkat, S., Dorger, M., and Goetz, A.E. 2006. Role of P-selectin in platelet sequestration in pulmonary capillaries during endotoxemia. J. Vasc. Res. 43:473-481.

14. Kuebler, W.M., Parthasarathi, K., Wang, P.M., and Bhattacharya, J. 2000. A novel signaling mechanism between gas and blood compartments of the lung. J. Clin. Invest. 105:905-913.

15. Andonegui, G., et al. 2003. Endothelium-derived Toll-like receptor- 4 is the key molecule in LPSinduced neutrophil sequestration into lungs. J. Clin. Invest. 111:1011-1020. doi:10.1172/JCI200316510.

16. Silliman, C.C., et al. 2003. Plasma and lipids from stored platelets cause acute lung injury in an animal model. Transfusion. 43:633-640.

17. The ARDS Network. 2000. Ketoconazole for early treatment of acute lung injury and acute respiratory distress syndrome: a randomized controlled trial. JAMA. 283:1995-2002.

18. Pitchford, S.C., et al. 2003. Platelets are essential for leukocyte recruitment in allergic inflammation. J. Allergy Clin. Immunol. 112:109-118.

19. O'Sullivan, B.P., et al. 2005. Platelet activation in cystic fibrosis. Blood. 105:4635-4641.

20. Pitchford, S.C., et al. 2005. Platelet P-selectin is required for pulmonary eosinophil and lymphocyte recruitment in a murine model of allergic inflammation. Blood. 105:2074-2081.

\title{
Proinsulin: a unique autoantigen triggering autoimmune diabetes
}

\author{
Sylvaine You and Lucienne Chatenoud
}

Université René Descartes Paris 5, INSERM U580, Hôpital Necker-Enfants Malades, Paris, France.

\begin{abstract}
In healthy individuals the immune system does not react aggressively toward host cells, a phenomenon defined as self tolerance. If self tolerance is broken autoimmune disease can develop, during which autoreactive lymphocytes are directed to a variety of autoantigenic epitopes. However, researchers have yet to determine whether immune responses to multiple autoantigens develop independently of each other or are the result of the response "spreading" from one autoantigen to another. In a study of NOD mice in this issue of the JCI, Krishnamurthy et al. show that the autoreactive $\mathrm{T}$ cell response to the autoantigen proinsulin lies upstream of that to islet-specific glucose-6phosphatase catalytic subunit-related protein, suggesting that the pathogenic autoimmune response to proinsulin subsequently spreads to other antigens (see the related article beginning on page 3258). These data support the current view that this pancreatic $\beta$ cell hormone is the first autoantigen targeted by the immune response in autoimmune diabetes.
\end{abstract}

Nonstandard abbreviations used: IGRP, islet-specific glucose-6-phosphatase catalytic subunit-related protein.

Conflict of interest: The authors have declared that no conflict of interest exists.

Citation for this article: J. Clin. Invest. 116:3108-3110 (2006). doi:10.1172/JCI30760.
Pathologic autoimmunity is characterized by an aberrant, self-perpetuating, immune-mediated, inflammatory response. It is the uncontrolled chronicity of this response that eventually leads to irreversible destruction of the target tissue. Among the major mechanisms under- lying this chronicity is the diversification of the pathogenic autoimmune response, also termed epitope spreading.

The concept of epitope spreading was initially described by Eli Sercarz in the early 1990s in autoantigen-induced EAE, which is a model for multiple sclerosis (1). This term was used to describe how a self-directed immune response induced by a single peptide (or epitope) could spread to include other peptides (or epitopes) not only on the same autoantigen (i.e., intramolecular spreading), but also on other self molecules clustered in close vicinity within the target cell (i.e., intermolecular spreading). Thereafter, several studies confirmed the crucial role of epitope spreading in EAE (2-4) and also in demyelinating diseases of the central nervous system that follow some viral infections (e.g., Theiler's murine encephalomyelitis; ref. 5) and IDDM, also known as type 1 diabetes $(6,7)$. 
A Epitope spreading

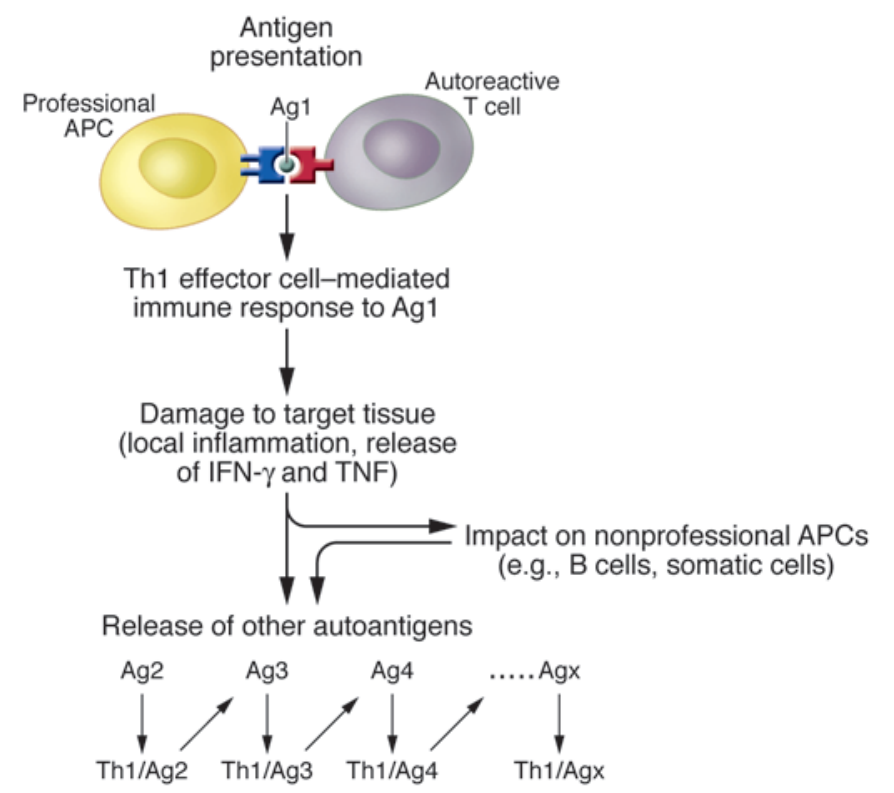

B Bystander suppression

Induction of tolerance to $\mathrm{Ag} 1$ (via oral, i.v., intranasal, s.c. delivery)

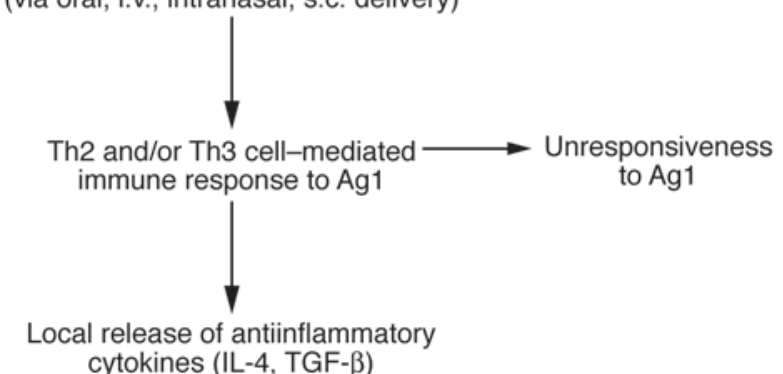

Downregulation of immune responses to other autoantigens

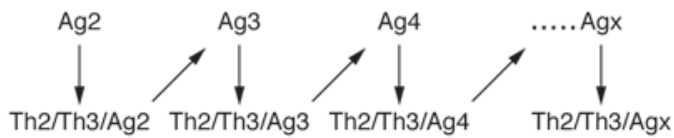

Figure 1

Epitope spreading versus bystander suppression. (A) In the event that an autoimmune response is triggered by a primary autoantigen (Ag1), the cytokine-mediated proinflammatory environment favors first the development of a Th1 effector cell-mediated immune response to Ag1 (Th1/Ag1), then the release from the damaged target tissue of other autoantigens (Ag2, Ag3, etc.), which trigger specific responses. This spread of specificity of the autoimmune response is one major molecular basis for its chronicity. (B) Bystander suppression operates when self tolerance is induced to one of the candidate autoantigens. The antiinflammatory environment generated may in turn downregulate the autoimmune responses to the other autoantigens involved in the autoimmune response. Major cytokines participating in this antiinflammatory effect are IL-4 and TGF- $\beta$, which are produced by Th2 and Th3 regulatory cells, respectively.

However, a central question remains as to whether an autoantigen triggers a primary insult, causing the release of other autoantigens from the damaged target cell in the context of a proinflammatory environment, and thus promotes the subsequent immune responses.

\section{Epitope spreading in autoimmune diabetes: from proinsulin to other islet antigens}

This crucial question is addressed by Krishnamurthy et al. in this issue of the JCI using a set of sophisticated in vivo experimental tools in a mouse model of autoimmune diabetes (8). The authors directly demonstrate that epitope spreading plays a role in a central loop that amplifies the autoimmune process, leading to disease chronicity. In addition, they provide strong and clear evidence in support of proinsulin being the primary autoantigen.

The analysis focused on 2 major autoantigens involved in type 1 diabetes: proinsulin 2 and islet-specific glucose6-phosphatase catalytic subunit-related protein (IGRP). Pathogenic T cells specific for these 2 antigens are present in signifi- cant numbers in infiltrated islets of NOD mice (which develop spontaneous IDDM) and exhibit efficient diabetogenic properties, as demonstrated by their capacity to transfer disease to immunoincompetent syngeneic recipients (9-13). Two different transgenic animal models were analyzed: NOD-PI mice, which overexpress proinsulin 2 in their APCs (14), and NOD-IGRP mice, which overexpress IGRP in their APCs. While the 2 transgenic mouse lines were fully tolerant to the autoantigen they overexpressed, they exhibited quite a different response in terms of disease. NOD-PI mice were insulitis and diabetes free as well as completely deficient of IGRP-reactive T cells. In contrast, NOD-IGRP mice were not protected from disease in spite of being tolerant to IGRP, as shown by the total absence of IGRP-specific $\mathrm{CD}^{+}$cytotoxic $\mathrm{T}$ cells. In fact, these animals exhibited an anti-proinsulin-autoreactive response that was identical to that observed in conventional NOD mice (8).

These data support the conclusion that the immune responses to IGRP lie downstream of those to proinsulin and are tightly dependent on the generation of a primary anti-proinsulin response. Proinsulin has long represented an ideal "primary" candidate for triggering autoimmune diabetes based on its highly restricted expression in pancreatic $\beta$ cells. However, until recently only indirect evidence had accumulated in support of such a conclusion. The recent data from Eisenbarth and colleagues (15) represented the first direct demonstration that, in NOD mice, part of the sequence of the $\mathrm{B}$ insulin chain is a primary target of the immune response. NOD mice lacking native insulin genes and carrying a mutated proinsulin transgene do not develop insulin autoantibodies, insulitis, or diabetes (15). In contrast, autoimmunity develops in mice carrying even a single copy of the native insulin gene (15). The study by Krishnamurthy et al. (8), via the use of a different experimental approach, provides additional proof for such a key role of proinsulin.

\section{What cellular and molecular factors propagate the spread?}

The study by Krishnamurthy et al. provides important clues regarding the initiation of epitope specificity and epitope dominance 
as well as the hierarchy of the immune responses to autoantigens in type 1 diabetes (8). However, important questions concerning the cellular and molecular events that initiate and perpetuate epitope spreading remain to be addressed.

At least 3 distinct factors may be involved: the nature of the antigenic determinant, the cytokines present in the milieu, and the type of APC involved. One important implication of the epitope spreading phenomenon is that, at least in the case of intramolecular spreading, subdominant or cryptic epitopes (i.e., not normally "seen" by the immune system) become "visible" and thus contribute to the autoimmune response. The type of cytokine present in the environment is also a key element. In particular, high levels of IFN- $\gamma$ produced by pathogenic $\mathrm{CD}^{+}$Th 1 cells enhance target cell immunogenicity by upregulating $\mathrm{MHC}$ and costimulatory molecules at the surface of APCs and somatic cells. In addition, a number of reports highlight the essential role of nonprofessional antigen presentation (i.e., mediated by cells other than dendritic cells, the professional APCs) in perpetuating autoimmune responses. In the peptide- or Theiler virus-induced EAE model, microglial cells resident in the CNS function as efficient APCs capable of activating $T$ cells and contributing to epitope spreading $(16,17)$. Similarly, in a model of the autoimmune disease myasthenia gravis, presentation of an epitope of the acetylcholine receptor by myoblasts favors spreading of the immune response (18). Lastly, autoreactive $B$ cells were shown to be strongly involved in the diversification of autoimmune $\mathrm{T}$ cell responses. Thus, during the course of autoimmune thyroiditis, autoantibodies to thyroglobulin could alter antigen processing, favoring the presentation of subdominant pathogenic epitopes (19). In type 1 diabetes, NOD mice deprived of $B$ cells are disease and insulitis free (20). It has also been shown that B lymphocytes can process $\beta$ cell autoantigens captured by surface immunoglobulins and present them to $\mathrm{T}$ cells contributing to the maintenance of the autoimmune response (21-23).

Therefore, professional APCs such as dendritic cells may be required to initiate the autoimmune reaction by adequately processing and presenting the primary autoantigen to naive autoreactive $T$ cells. Once activated, these autoreactive effectors create a proinflammatory environment that in turn influences and modifies the behavior of other cell types, including immune or somatic cells, to acquire APClike functional properties (Figure 1).

The experimental model described by Krishnamurthy et al. (8) represents an interesting tool with which to obtain further insights into these issues, which are important from both fundamental and therapeutic points of view. In fact, the molecular processes that spread pathogenic responses are probably similar to those operating to spread protection in the case of bystander suppression $(24,25)$. It has been well established in different models of autoimmunity, including EAE (24) and type 1 diabetes, that protection from disease may be induced following delivery by various routes of any of the candidate autoantigens (in the case of autoimmune diabetes using proinsulin, insulin, heat shock protein 60, or glutamic acid decarboxylase). Results of mechanistic studies confirmed that the effective downregulation of the immune response specific to the therapeutic autoantigen rapidly extends to other candidate autoantigens $(24,25)$ (Figure 1). A better understanding of the underlying molecular mechanisms of these interactions will be essential to expedite the transfer of autoantigen therapy to the clinic.

Address correspondence to: Lucienne Chatenoud, INSERM U580, Hôpital Necker-Enfants Malades, 161 Rue de Sèvres, 75015 Paris, France. Phone: 33144-49-53-73; Fax: 33-143-06-23-88; E-mail: chatenoud@necker.fr.

1. Lehmann, P.V., Forsthuber, T., Miller, A., and Sercarz, E.E. 1992. Spreading of T-cell autoimmunity to cryptic determinants of an autoantigen. Nature. 358: $155-157$.

2. Yu, M., Johnson, J.M., and Tuohy, V.K. 1996. A predictable sequential determinant spreading cascade invariably accompanies progression of experimental autoimmune encephalomyelitis: a basis for peptide-specific therapy after onset of clinical disease. J. Exp. Med. 183:1777-1788.

3. Tuohy, V.K., Yu, M., Weinstock-Guttman, B., and Kinkel, R.P. 1997. Diversity and plasticity of self recognition during the development of multiple sclerosis. J. Clin. Invest. 99:1682-1690.

4. Tuohy, V.K., et al. 1998. The epitope spreading cascade during progression of experimental autoimmune encephalomyelitis and multiple sclerosis. Immunol. Rev. 164:93-100.

5. Miller, S.D., et al. 1997. Persistent infection with Theiler's virus leads to CNS autoimmunity via epitope spreading. Nat. Med. 3:1133-1136.

6. Tisch, R., et al. 1993. Immune response to glutamic acid decarboxylase correlates with insulitis in nonobese diabetic mice. Nature. 366:72-75.

7. Kaufman, D.L., et al. 1993. Spontaneous loss of T-cell tolerance to glutamic acid decarboxylase in murine insulin-dependent diabetes. Nature. 366:69-72.
8. Krishnamurthy, B., et al. 2006. Responses against islet antigens in NOD mice are prevented by tolerance to proinsulin but not IGRP. J. Clin. Invest. 116:3258-3265. doi:10.1172/JCI29602.

9. Wegmann, D.R., Norbury-glaser, M., and Daniel, D. 1994. Insulin-specific T cells are a predominant component of islet infiltrates in pre-diabetic NOD mice. Eur. J. Immunol. 24:1853-1857.

10. Daniel, D., Gill, R.G., Schloot, N., and Wegmann, D. 1995. Epitope specificity, cytokine production profile and diabetogenic activity of insulin-specific $\mathrm{T}$ cell clones isolated from NOD mice. Eur. J. Immunol. 25:1056-1062.

11. Verdaguer, J., et al. 1997. Spontaneous autoimmune diabetes in monoclonal $\mathrm{T}$ cell nonobese diabetic mice. J. Exp. Med. 186:1663-1676.

12. Lieberman, S.M., et al. 2003. Identification of the beta cell antigen targeted by a prevalent population of pathogenic CD8+ T cells in autoimmune diabetes. Proc. Natl. Acad. Sci. U. S. A. 100:8384-8388.

13. Utsugi, T., et al. 1996. Major histocompatibility complex class I-restricted infiltration and destruction of pancreatic islets by NOD mouse-derived beta-cell cytotoxic CD8(+) T-cell clones in vivo. Diabetes. 45:1121-1131.

14. French, M.B., et al. 1997. Transgenic expression of mouse proinsulin II prevents diabetes in nonobese diabetic mice. Diabetes. 46:34-39.

15. Nakayama, M., et al. 2005. Prime role for an insulin epitope in the development of type 1 diabetes in NOD mice. Nature. 435:220-223.

16. McMahon, E.J., Bailey, S.L., Castenada, C.V., Waldner, H., and Miller, S.D. 2005. Epitope spreading initiates in the CNS in two mouse models of multiple sclerosis. Nat. Med. 11:335-339.

17. Vanderlugt, C.L., and Miller, S.D. 2002. Epitope spreading in immune-mediated diseases: implications for immunotherapy. Nat. Rev. Immunol. 2:85-95.

18. Curnow, J., Corlett, L., Willcox, N., and Vincent, A. 2001. Presentation by myoblasts of an epitope from endogenous acetylcholine receptor indicates a potential role in the spreading of the immune response. J. Neuroimmunol. 115:127-134.

19. Dai, Y., et al. 1999. Enhancing or suppressive effects of antibodies on processing of a pathogenic T cell epitope in thyroglobulin. J. Immunol. 162:6987-6992.

20. Serreze, D.V., et al. 1996. B lymphocytes are essential for the initiation of $\mathrm{T}$ cell- mediated autoimmune diabetes: analysis of a new "speed congenic" stock of NOD.Ig mu(null) mice. J. Exp. Med. 184:2049-2053.

21. Serreze, D.V., et al. 1998. B lymphocytes are critical antigen-presenting cells for the initiation of $\mathrm{T}$ cell-mediated autoimmune diabetes in nonobese diabetic mice. J. Immunol. 161:3912-3918.

22. Reijonen, H., Daniels, T.L., Lernmark, A., and Nepom, G.T. 2000. GAD65-specific autoantibodies enhance the presentation of an immunodominant T-cell epitope from GAD65. Diabetes. 49:1621-1626.

23. Tian, J., Zekzer, D., Lu, Y., Dang, H., and Kaufman, D.L. 2006. B cells are crucial for determinant spreading of $\mathrm{T}$ cell autoimmunity among beta cell antigens in diabetes-prone nonobese diabetic mice. J. Immunol. 176:2654-2661.

24. Al Sabbagh, A., Miller, A., Santos, L.M., and Weiner, H.L. 1994. Antigen-driven tissue-specific suppression following oral tolerance: orally administered myelin basic protein suppresses proteolipid protein-induced experimental autoimmune encephalomyelitis in the SJL mouse. Eur. J. Immunol. 24:2104-2109.

25. Tian, J., Lehmann, P.V., and Kaufman, D.L. 1997. Determinant spreading of T helper cell 2 (Th2) responses to pancreatic islet autoantigens. J. Exp. Med. 186:2039-2043. 


\title{
The sticky truth about angiogenesis and thrombospondins
}

\author{
Judith A. Varner \\ Moores UCSD Cancer Center, La Jolla, California, USA.
}

\begin{abstract}
The formation of new blood vessels, a process known as angiogenesis, is important for embryonic development and wound healing as well as the development of cancer and inflammation; therefore, angiogenesis is a valuable target for clinical intervention. Both logic and empiricism suggest that a balance of stimulatory and inhibitory switches is required for orderly formation of blood vessels. Thrombospondins 1 and 2 were among the first natural angiogenesis inhibitors to be identified. However, the cellular origins and mechanisms of action of these important proteins during angiogenesis have remained largely unknown. Studies by Kopp et al., presented in this issue of the JCI, clarify some of these issues by revealing that megakaryocytes and their "sticky" wound-healing progeny, platelets, are important sources of thrombospondins 1 and 2 and that these thrombopoietic cells play key roles in controlling blood vessel formation during hematopoiesis and ischemic wound healing (see the related article beginning on page 3277 ).
\end{abstract}

Blood vessels convey the 3 essential elements of life (food, water, and air, or oxygen) to all tissues. The development of these conduits of life-giving essentials allowed the evolution of large organisms with complex tissues but required precise control mechanisms - mechanisms that are often perturbed during disease progression.

Identification of the systems controlling the formation of blood vessels has been the subject of over 30 years of intensive research. Studies of normal angiogenesis, such as occurs during wound healing, suggest that it is a finely regulated, short-term process that is initiated by the release of stimulatory factors from platelets, "sticky" circulating cells that plug wounds in blood vessel walls or stromal cells, such as fibroblasts. New vessel formation is then inhibited by the absence of stimulatory factors and/or by naturally arising inhibitory factors that may include proteolytic fragments of extracellular matrix proteins. Early developments in the field of angiogenesis research led to the discovery of many angiogenesis-stimulating factors, including the well-known VEGF, and to the discovery of naturally occurring angiostatic agents,

Nonstandard abbreviations used: SDF-1, stromal cell-derived factor 1; TSP, thrombospondin; TSP-DKO, TSP double-knockout (mice).

Conflict of interest: The author has declared that no conflict of interest exists.

Citation for this article: J. Clin. Invest. 116:3111-3113 (2006). doi:10.1172/JCI30685. such as the thrombospondins (TSPs) (1-3). TSP1 was the first naturally arising angiogenesis inhibitor to be described (1-3). Shortly thereafter, a second TSP, TSP2, was also found to inhibit angiogenesis (4). Although much is known about these natural angiostatic proteins, much remains uncertain. For example, it has not been clear which cells express TSP1 and TSP2 during angiogenesis in vivo. The exact mechanisms by which TSPs inhibit angiogenesis in vivo have also remained elusive. The studies by Kopp et al. in this issue of the JCI shed new light upon these mysteries (5).

\section{TSP1 and TSP2}

TSPs are a family of 5 structurally related extracellular matrix proteins with the capacity to bind both cell surface receptors and other extracellular matrix proteins (6). TSP1 and TSP2 are very similar structurally and functionally; both have been implicated as inhibitors of angiogenesis, endothelial cell survival, and endothelial cell migration (7). TSP1, the first of the family to be described, is a thrombin-sensitive extracellular matrix protein that is released from platelet granules after activation (8). Importantly, it is the most abundant protein in platelet $\alpha$-granules. It was isolated from platelets and was localized to megakaryocytes and the extracellular matrix as early as $1978(8,9)$. It is also expressed by endothelial cells and fibroblasts in vitro and by certain tumor cells in vitro and in vivo $(10,11)$. A similar protein, TSP2, was identified in endothelial cells a few years later (4). TSP1 is an arginine-glycine-aspartic acid-containing ligand for integrin $\alpha v \beta 3$ (6). It is also a ligand for CD36, a G protein-coupled receptor on endothelial cells (6). Several studies indicate that TSP1 binding to CD36 inhibits endothelial cell migration and induces endothelial cell apoptosis in vitro (12-15). TSP1 also binds to other ECM components, such as fibrinogen, fibronectin, and proteoglycans, and plays a role in platelet aggregation (16).

In 1990, TSP1 was first described as an inhibitor of angiogenesis, as it blocked the formation of new blood vessels in the cornea in vivo in response to basic FGF $(1,3)$ and blocked endothelial cell tube formation and cell migration in vitro (2). Additional studies showed that TSP1 could serve as a naturally occurring tumor suppressor by inhibiting tumor angiogenesis (3). Recent studies indicate that loss of TSP1 or TSP2 during embryonic development is not lethal but results in increased vascular density $(17,18)$. Various studies indicate that adult TSP2 $2^{-/-}$and $T S P 1^{-/-}$mice exhibit increased vascular density during wound healing, retinal development, and tumor growth $(17,18)$. In contrast, TSP1 transgenic mice exhibit decreased vascular density (19).

\section{What is the source of thrombospondins?}

The tissue source of TSP during angiogenesis has been unclear. During embryonic development, TSP2, but not TSP1, is expressed in endothelia and developing connective tissues (20). In contrast, TSP1, but not TSP2, is expressed in megakaryocytes and in the developing kidney and gut (20). Other studies have shown that TSP1 is expressed by megakaryocytes and platelets in the adult animal $(8,9)$ and that both TSPs are expressed in endothelial cells cultured in vitro $(2,10,11)$. Fibroblasts also can express TSP1 and TSP2 (1, 21). As endothelial cells, fibroblasts, and platelets are all present in healing wounds and tumors, it has not been clear which cell 